\title{
Corela
}

Cognition, représentation, langage

16-1 | 2018

Vol. $16, \mathrm{n}^{\circ} 1$

\section{La formation des locutions conjonctives : Le cas de lorsque}

\section{Leïla Ben Hamad}

\section{CpenEdition}

\section{Journals}

\section{Édition électronique}

URL : http://journals.openedition.org/corela/5354

DOI : $10.4000 /$ corela. 5354

ISSN : $7638-573 X$

\section{Éditeur}

Cercle linguistique du Centre et de l'Ouest - CerLICO

\section{RÉFÉRENCE ÉLECTRONIQUE}

Leila Ben Hamad, "La formation des locutions conjonctives : Le cas de lorsque », Corela [En ligne], 16-1 | 2018, mis en ligne le 28 juin 2018, consulté le 19 avril 2019. URL : http://

journals.openedition.org/corela/5354;DOI : 10.4000/corela.5354

Ce document a été généré automatiquement le 19 avril 2019

\section{(c) (i) (9) (2)}

Corela - cognition, représentation, langage est mis à disposition selon les termes de la licence Creative Commons Attribution - Pas d'Utilisation Commerciale - Partage dans les Mêmes Conditions 4.0 International 


\title{
La formation des locutions conjonctives : Le cas de lorsque ${ }^{1}$
}

\author{
Leïla Ben Hamad
}

1 Nous avons pour ambition de fournir une analyse longitudinale de l'émergence et de l'évolution des locutions conjonctives sur plus d'un millénaire de langue française. Nous nous pencherons ici sur le cas de lorsque. Nous nous occuperons de décrire les processus qui l'ont conduit à entrer dans la grammaire du français. C'est une partie importante (autant du point de vue morphologique, syntaxique que sémantique) de l'histoire de la langue qui sera en jeu.

2 La première partie, consacrée à l'étude de cette locution conjonctive dans le premier état $\mathrm{du}$ français écrit, a pour objet de mettre en perspective son émergence. L'enjeu est de reconstituer finement cette émergence et de découvrir le processus qui y est sous-jacent.

3 La deuxième partie examinera la locution, nouveau-née, sous l'angle de l'évolution. Une longue quête procèdera à mettre en lumière des phénomènes de changements isochrones et de variations concomitantes et à en proposer une analyse théorique, afin de cerner la nature des processus qui y sont impliqués. Les bases informatisées BFM, DMF et Frantext nous auront fourni le champ d'une recherche suivie sur une période qui couvrira la langue française, dès le premier état du français écrit jusqu'au français d'aujourd'hui.

4 Enfin, dans une troisième et dernière partie, nous proposerons une analyse théorique des phénomènes en jeu dans la formation des locutions conjonctives : quelle est la part de la grammaticalisation, et quelle est celle de la pragmaticalisation?

\section{L'émergence de lors que}

$5 \quad$ Nous étudierons ici les processus impliqués dans l'apparition de lors que, qui s'intègre dans un paradigme naissant, celui des locutions conjonctives de simultanéité. 


\subsection{L'apparition de lors que dans les textes}

6 Dans les tout premiers textes français, il n'y a aucune occurrence de lors que. C'est dans Erec et Enide de Chrétien de Troyes, composé dans le domaine champenois vers 1170, qu'on le rencontre pour la première fois ${ }^{2}$ :

(1) M'an voldrai par matin aler,

Lors que le jor verrai lever. (Chrétien de Troyes, Erec et Enide, v. 4220 ; vers 1170)

(2) Sovant plore, sovant sopire,

Molt li est po de son empire

Et de la grant enor qu'ele a,

Lores que Cligés s'en ala. (Chrétien de Troyes, Cligès, v. 4315-4318; vers 1176)

(3) Et lors que ele pot veoir

Le roi, si leissa jus cheoir (Chrétien de Troyes, Chevalier au Lion ou Yvain, v. 2713-2714 ;

vers 1177 et 1181)

On peut considérer que cette innovation relève de l'idiolecte de l'auteur d'autant que c'est chez ce même auteur qu'on rencontre les premiers emplois de a ce que et que que $e^{3}$, qui en viennent à exprimer une notion fort éloignée du sens originel de leurs composantes ${ }^{4}$ :

(4) A ce que je puis m'an tandrai,

Et le sairemant an prandrai,

Que vers moi si vos contendroiz (Chrétien de Troyes, Chevalier de la Charrette ou Lancelot

, v. 5491-5493; entre1177 et 1181)

(5) Que qu'il chancele, Erec le bote,

Et cil chiét sor le destre cote (Chrétien de Troyes, Erec et Enide, v. 983-984 ; cf. aussi, v. 2795,6327 ; vers 1170)

8 Il y a aussi lieu de considérer l'intégration de lors que dans le paradigme des locutions conjonctives de simultanéité comme étant de caractère analogique, sur le modèle des autres constructions en que attestées dans d'autres textes, à peu près contemporains :

(6) Endementres qu'ele demeure

Vint un serpant de male part,

Issi du bois si vint el jart. (Roman de Thèbes, v 2366-2368; vers 1150)

(7) Entrues qu'il kaça la folie

Etioclés pas ne s'oblie. (Roman de Thèbes, v. 7073-7074 ; vers 1150)

(8) Cest ducheaume qu'il a tenu,

En pais tant dis qu'il a vescu. (Saint-Maure (de), Chronique des ducs de Normandie, II, v.

8561-8562 ; cité par Godefroy, $(1969$ : 637) ; 1160)

(9) Se [la Renommée] de la rien set tant ne quant,

De molt petit fait assez grant,

Ele l'acroist et plus et plus,

Quant qu'ele vait et sus et jus. (Eneas, v. 1543-1546; 1155)

9 Mais, selon le point de vue que l'on adopte, on est conduit à centrer différemment l'analyse.

La perspective habituelle, essentiellement morpho-syntaxique, voit dans la formation des locutions conjonctives une mutation du système grammatical, qui tend à l'uniformisation des réalisations discursives sous la forme de la structure base-que $P$. On invoque ainsi l'analogie comme facteur justifiant la réfection d'une forme adverbiale (ou prépositionnelle) sur le modèle d'une «forme de fondement ${ }^{5}$ » en que, sentie comme régulière. 
11 En revanche, si l'on se place, comme nous le ferons ici, dans une perspective sémanticocognitive, la question est de savoir ce qui rend possible la création d'une nouvelle locution, et en particulier à quels déplacements de sens elle doit son émergence. Nous procéderons ici à l'analyse précise du contexte qui a favorisé l'émergence de lorsque. Nous tenterons de formuler des hypothèses ${ }^{6}$ sur la nature des opérations dont ce contexte peut être la trace.

Le premier constat que l'on peut faire - à partir des données de corpus dont nous disposons - est que les «locutions conjointes primaires ${ }^{7}$ » articulées sur que sont bien attestées à date ancienne, à côté de divers autres agencements binaires.

Dementres que est déjà assez bien établi au moment où apparaît une importante production littéraire en langue « vulgaire $»^{8}$ :

(10) Que alquune fiede ne ravisset sicume leuns la meie aneme, dementres que n'est chi reaimet ne chi salf facet. (Libri psalmorum : 6 ; première moitié du XII ${ }^{\text {èmesiècle) }}$

(11) Dementres que s'enorguilist li fel, est espris li povre. (Psautier de Cambridge : 9, cité par Graeme-Ritchie (1907: 83); début XII ${ }^{\text {ème }}$ siècle)

15 La «vieille langue » a aussi eu recours à por tant que, qui marque la simultanéité accompagnant la durée du procès principal jusqu'à son terme, i.e. exprime une nuance particulière dans la simultanéité :

(12) (...) ne teiz nen iert jamais, por tant ke secles vive (Li ver del Juise, v.10 ; cité par Graeme-Ritchie (op. cit. : 83) ; début XII ${ }^{\text {ème }}$ siècle)

16 On ne peut donc parler d'une nouvelle tendance, allant d'une forme-base à une formedérivée en que. Aussi, n'avons-nous pas d'emploi attesté de lors (élément de base ou élément-source de lorsque) avant 1130, dans Gormont et Isembart :

(13) (...), e vit Gormund el pui ester :

Si lors ne vait a lui juster,

Dunc se tendra pur afolé. (Gormont et Isembart, v-116-118; vers 1130)

17 Quel est dans ce cadre le statut qu'il faut accorder à lors que? Si ce n'est pas une forme analogique, alignée sur les formes-modèles préexistantes ou "formes de fondement », estce une forme nouvelle, résultat d'une "transformation innovante ${ }^{9}$ ? Est-il fondé de le traiter ainsi ?

18 Nous prêterons ici attention aux prémices de lors que. Nous procéderons à l'analyse précise du contexte grammatical où se situe ce morphème naissant. Nous examinerons notamment la variation qui repose sur l'existence d'une triple série de formes pendant la période du français médiéval. De ce point de vue, c'est l'ensemble des formes concernées qui doit nous interpeller puisque lors que s'impose successivement comme forme parallèle, comme alternative ou comme substitut ${ }^{10}$.

\subsection{Les prémices de lors que}

Pendant la seconde moitié du XII ${ }^{\text {ème }}$ siècle, on constate un afflux considérable de formes analytiques qui concurrencent largement les formes synthétiques, voire les supplantent ${ }^{11}$. Ces formes analytiques laissent aux éléments de base la responsabilité d'identifier la valeur précise de la locution subordonnante ${ }^{12}$, tendant à imposer une large homogénéité ${ }^{13}$ - Celles-ci ne fonctionnent pas de façon compacte et inanalysable comme les formes synthétiques correspondantes d'autant qu'elles ne sont pas des constructions primaires mais le produit de restructurations syntactico-sémantiques ${ }^{14}$. Elles apparaissent comme la conjonction de deux opérations : le marquage de la coïncidence (cum/com(e), quant, que) 
d'une part et le marquage d'une certaine nuance grâce à l'élément de base adjacent (lors, or, $s i, ..)^{15}$ de l'autre. Dans ces constructions, se discerne quelque chose de nouveau, le lien privilégié qu'entretient le subordonnant avec le composant-base. C'est le résultat d'une tendance qui émerge dès les débuts du XII ${ }^{\text {mè }}$ siècle, à une spécialisation de plus en plus stricte des formes.

\subsubsection{Développement de la forme lors quant : une locution 'récente'}

Notre corpus présente un large éventail de locutions à base de quant, qui se sont développées dans les chansons de gestes et les traductions de la première moitié du XII ${ }^{\text {ème }}$ siècle. On constate notamment l'extension progressive des emplois de lors quant, qui se rencontre pour la première fois- à notre connaissance- chez Philippe de Thaon dans le Comput et le Bestiaire, composés en Angleterre entre 1113 (ou 1119) et 1121 (ou 1135) :

(14) E tuit icil del munt

Ki veir crestïen sunt

Lores quant finerunt

Altresi Dé verunt. (Philippe de Thaon, Bestiaire, v. 2089-2092 ; cf. aussi v. 131, 1846 ;

entre 1121 et 1135)

Cette locution, qui semble être une particularité de l'auteur au début du XII ${ }^{\text {ème }}$ siècle, s'est particulièrement développée à la fin du siècle, dans diverses aires régionales et dans divers types de textes :

(15) Doiez dire moi ne autrui,

S'il i fu lors quant je i fui

- Oïl, mes ne le coneüstes.

Il i fu lors quant vos i fustes,

Et avoec vos s'an departi. (Chrétien de Troyes, Cligés, v. 5154-5157; 1176)

(16) Prez sui que je li face soudre,

Lors qant Renart sera venuz

Et li jugemenz iert tenuz ; (...) (Roman de Renart, Branche I, v. 120-122 ; 1180)

Parallèlement, se développent aussi les formes analytiques à base nominale, et notamment le tour l'ore que ${ }^{16}$.

\subsubsection{Le développement de l'ore que : un autre processus de changement}

23 Nombreux sont les emplois de l'ore que dans les textes médiévaux, et notamment pendant la deuxième moitié du XII ${ }^{\text {ème }}$ siècle, en cette période cruciale de l'entrée de lors que dans la grammaire du français.

(17) L'ore que charrete fu primes

Pansee et feite soit maudite ;

Car molt est vix chose et despite. (Chrétien de Troyes, Chevalier de la Charrette ou

Lancelot, v. 2608-2610; vers 1177 et 1181)

Ces emplois sont intéressants diachroniquement, car ils sont très anciennement attestés :

(18) Si li requerent conseil d'icele chose

Qu'il unt oït, ki mult les desconfortet

Ne guardent l'ure que terre nes enclodet. (Vie de saint Alexis, v. 304-305; vers 1050)

L'ore que est de formation pré-littéraire. Elle apparaît pour la première fois, nous semblet-il, dans Sant Lethgier composé vers l'an mil : 
(19) Cil Laudeberz, qual hora 1 vid

Torne s'als altres, si llor dist (Sant Lethgier, v. 204-205, cf. aussi v. 149 ; seconde

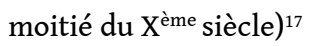

et prend de l'ampleur dès la première moitié du XII ${ }^{\text {ème }}$ siècle :

(20) De cest cheval que j'ai ci guaaignié !

Or nel donreie por l'or de Montpelier

Ui fu tel ore que molt l'oi coveitié. (Couronnement de Louis, v. 1147-1150; vers

1130)

(21) Bone fud l'ore que le suen cors fud né !

Plus de treis mil lur en ad mort jeté. (Chanson de Guillaume, v. 3147-3148; vers 1140)

Notons que que se montre ici comme le siège d'une relation anaphorique et présente ainsi un fonctionnement analogue à celui des relatifs ${ }^{18}$. Ce qui est tentant d'un point de vue non seulement structural mais également sémantique. Que permet de reprendre en anaphore le substantif temporel. La proposition qu'il introduit sert ainsi à en expliciter et à en développer le contenu.

Il est aussi envisageable d'interpréter quant comme un relatif dans lors quant, c'est-à-dire comme une reprise anaphorique d'une source ${ }^{19}$. Quant représente un relais sémique ou encore assure un lien de congruence sémique avec le sémantème de l'élément de base (ou l'élément source) lors. évidente) dans :

(22) M'an voldrai par matin aler,

Lors que le jor verrai lever. (Chrétien de Troyes, Erec et Enide, v. 4220 ; vers 1170)

C'est cette reprise anaphorique qui, par la même occasion, assure la connexion entre les deux prédications. Que insère dans la proposition qu'il introduit la source adverbiale utile au cadrage de l'événement raconté. Voilà qui plaide en faveur de son appréhension en tant que proforme relative, ce qui implique le réalignement de son rôle sur celui qu'il remplit dans le tour à base nominale. À preuve, d'une part, le fait qu'un nom temporel est à l'origine de l'adverbe-source ${ }^{20}$ et d'autre part, le fait que les adverbes donc, adonc, idonc et atant, qui ne comprennent pas dans leur formation un nom de temps, pouvant être intégré dans une structure relative, ne permettent pas la construction en que $P$.

31 Par ailleurs, que représente anaphoriquement le mot-source dans l'élément phrastique qu'il introduit, ce qui nous permet de faire l'analogie avec quant.

Dans une perspective diachronique, force est de constater que si lors que n'est pas issu historiquement de l'ore que, il en est du moins l'aboutissement logique. Il semble avoir été engendré, par rapprochement des deux premiers éléments, pouvant dès lors s'employer de manière autonome. Il s'avère du reste concevable, nous semble-t-il, d'émettre l'hypothèse que ce nouveau morphème se présente comme un « doublet » du morphème lors quant, existant depuis le début du XII ${ }^{\text {ème }}$ siècle et qui exprime déjà la même notion de simultanéité.

D'après le nombre de textes dont nous disposons, il semble qu'on ne puisse pas vraiment, par contre, distinguer des états successifs. Il est possible donc de conclure que ces constructions n'ont pas émergé les unes après les autres mais bien au contraire parallèlement. 


\section{Processus d'évolution de lorsque}

\subsection{Le développement de lors que}

Le développement de lors que s'effectue en raison inverse de la disparition totale de lors quant. Ce phénomène en croise un autre de même envergure. L'adverbe lors connaît une extension progressive de ses emplois. Nous examinerons ici le processus de remplacement de l'ancien morphème par la nouvelle locution. Nous nous attacherons notamment à mettre en évidence l'évolution de la répartition de leurs fréquences.- grâce à des précisions chiffrées ${ }^{21}$. Nous envisagerons ensuite les emplois diversifiés auxquels se prête l'élément de base lors.

\subsubsection{Lors quant et lors que : de la cooccurrence à la concurrence}

En ancien français jusqu'à la fin du XIII ${ }^{\text {ème }}$ siècle, lors que et lors quant n'étaient pas vraiment en concurrence. Il n'est, en effet, pas rare de les trouver en variation dans les mêmes textes :

(23) (...) Au meins celui qu'eles ont ores

(Si les pot il bien nomer lores

Quant il prumierement cria

Tout le monde et quan qu'il i a), (...). (Meun (de), Roman de la Rose, v.7075-7060;

entre 1269 et 1278)

(24) (...) Quant des mirouers parlions,

Don si briefmant nous passions,

Et de tout ce leur samble lores

Qu'il sait ainsinc por voir defores. (Meun (de), Roman de la Rose, v.18323-1826;

entre 1269 et 1278)

Un demi-siècle plus tard en revanche, le système aura basculé, lors que commençant à l'emporter. La cooccurrence lorsque et lors quant apparait de façon minoritaire encore dans les mêmes textes :

(25) Lors quant Remond ouy ces mos, si boute la table ensus de lui, et entre en sa chambre, espris de yre et de jalousie, et prent son espee qui pendoit a son chevez, et la ceint, et s'en va ou lieu ou il savoit bien que Melusigne s'en aloit tous les samedis, et treuve un fort huis de fer, moult espez, et sachiez de vray que oncques mais n'avoit esté si avant. (Jean d'Arras, Mélusine : $241 ; 1392-1393$ )

(26) Lors qu'il fu en la porte, si la commande a garder tant que ses hommes feussent venuz. (Jean d'Arras, Mélusine : $225 ; 1392-1393$ )

Le tournant des $\mathrm{XIV}^{\text {ème }} \mathrm{XVI}^{\text {ème }}$ siècles est la période où lors que entre vraiment en cooccurrence et/ou concurrence avec lors quant :

On assiste au déclin de lors quant dès le début du XV ème siècle. On observe, en outre, que sa fréquence d'emploi est peu élevée au XIVème. Aux XIV ${ }^{\text {ème }}$ et XV ${ }^{\text {ème }}$ siècles, dans le corpus du DMF, constitué d'environ 226 textes, en sont attestées près de 46 occurrences. Les auteurs du début du XVI ${ }^{\text {ème }}$ siècle connaissent et emploient encore lors quant, de loin en loin. On a recensé dans les 154 textes du XVI ${ }^{\text {ème }}$ siècle que regroupe Frantext, près de 18 occurrences de lors quant. Au XII ${ }^{\text {ème }}$ siècle, il n'est pas un texte de notre corpus qui en offre une seule occurrence.

Quant a donc été remplacé par que, après avoir alterné avec lui pendant près de cinq siècles. Il semblerait que ce phénomène de substitution soit le fruit du hasard ou le 
résultat d'un simple fait d'analogie. Cependant le fait qu'un tel bouleversement opère d'abord en moyen français, moment où cependant, pendant et durant commencent leur parcours d'accession au statut de éléments de base de locutions conjonctives, laisse penser qu'il se manifeste une tendance profonde à réaliser une neutralisation et une simplification du système. Ainsi, même si ces formes n'entrent que dans des locutions en que, elles participent néanmoins, d'une façon identique, au mouvement d'ensemble qui affecte la structure interne des locutions conjonctives. Par ailleurs, si l'on examine le phénomène dans son ensemble, on constate que ce changement structurel est en germe dès l'ancien français. Que connait, en effet, une extension progressive de ses emplois en articulation analytique entre la période la plus ancienne (IX ${ }^{\text {ème }}-\mathrm{XI}^{\text {ème }}$ siècles) et le moyen français (XIVème - XV ème siècles) ${ }^{22}$. La marginalisation de quant en tant que marqueur analytique n'est ni brutale ni seulement engendrée par le succès de que, par un effet mécanique en quelque sorte, mais elle est le produit d'une évolution qui s'est faite dans le sens de la simplification, de la spécialisation et de la clarté sémantique.

Nous nous appuierons ici sur la remarque de Imbs (op.cit.: 194-195) à propos de lors quant:

«Le premier élément est adverbial-nominal, suivant le modèle des propositions relatives les plus ordinaires; puis vient très régulièrement un élément relatif représenté par la consonne qu-, qui s'appuie sur cet antécédent; mais cet élément relatif est suivi d'un élément non relatif(-ant), qui lui est synthétiquement incorporé et qui ne fait que répéter l'idée exprimée par l'antécédent lors. »

41 Dans le cadre ainsi esquissé, quant possède un trait relationnel, certes, mais aussi référentiel, ce qui le rend redondant et ouvre ainsi la voie à son remplacement par que, l'« articulant vide de matière notionnelle » selon Jonas $(1971: 353)^{23}$. Que semble ainsi voué au rôle d'« un stabiliseur catégoriel ».

Il est un autre fait: lors ne peut se dispenser du que à partir du XVII ${ }^{\text {ème }}$ siècle $^{24}$. On discerne alors une certaine forme d'interdépendance qui n'est pas seulement forte d'un point de vue morpho-syntaxique mais s'impose aussi sur le plan sémantique.

On constate, par ailleurs, que le développement de lors que croise un autre changement, qui concerne l'extension remarquable de lors.

\subsubsection{Extension et diversification des emplois de lors}

C'est à partir du nom heure, issu de hora latin, et de l'article défini, qui a pour origine le démonstratif latin illa (distal, ou de troisième personne) ${ }^{25}$, que lors s'est - semble-t-il développé ${ }^{26}$. Après les phases pragmatiques de subjectivisation ${ }^{27}$ et de néo-analyse subséquente, le nouvel adverbe va voir ses emplois s'étendre à de nouveaux contextes, qui auraient été incompatibles avec le syntagme originel. Lors est essentiellement anaphorique ${ }^{28}:$ il montre rétroactivement qu'un cadre de discours s'est constitué, que certains éléments d'une situation ont été évoqués. Aussi n'est-il pas toujours aisé de déterminer ce à quoi lors sert de repère et, de ce fait, il est susceptible de se trouver dans la zone dite annexe :

(27) Se la cité fust lors assise

Ou alumee ou toute esprise,

$\mathrm{Ne}$ cuit que plus em plorissant

Ne greingnor deul en feïssant. (Roman de Thèbes, v. 1975-1978 ; vers 1150)

ou occuper la place de fondement ${ }^{29}$, qu'il soit précédé d'un coordonnant : 
(28) A tant se coise, a tant se tot

Cil qui a dolor sa vie use.

Mes lors cele qui a val muse,

Quan qu'il ot dit ot entandu ;

N'a plus longuemant atandu, (...) (Chrétien de Troyes, Cevalier de la Charrette ou

Lancelot, v.6530-6534; vers 1177-1181)

ou apparaisse à l'initiale absolue de l'énoncé :

(29) Ele l'esgarde, bien l'entent,

Vers lui se trait et mot ne dit.

Lors se tormente, lors s'ocit. (Narcisse, v. 976-978 ; vers 1165-1175)

Le lien anaphorique peut porter sur un contenu propositionnel équivalant à un seul membre de phrase ou à l'ensemble phrastique, qu'il appartient au lecteur (ou à l'interlocuteur) de repérer comme l'élément anaphorisé. La nature floue du lien anaphorique exprimé permet de relancer l'élan narratif ou encore de redonner une impulsion au récit ${ }^{30}$ :

(30) Lors la montent seur un palefroi et s'en vont en la forest la ou il la voient plus espesse. Et quant il furent bien enforesté, lors pranent garde s'il i sont tuit; si virent qu'il orent perdu trois de leur compaignons. Lors demande li uns a l'autre qu'il sont devenu. (ibid. : 125) (Mort le Roi Artu : $125 ; 1230$ )

Cet adverbe introduit dans le récit des événements un supplément de cohérence, de signification. Il contribue à en constituer la cohésion et en assurer le flux. Ce qui ne tient pas seulement à l'entité de la phrase ou du paragraphe anaphorisés, mais qui dépend aussi de la dimension de subjectivité qu'il introduit :

(31) Atant fine li parlemenz; si s'en revient li rois as tentes et enmeine avec soi la reïne. Lors commença entr'eus la joie si grant comme se Damledex i fust descenduz.

(Mort le Roi Artu : $120 ; 1230)^{31}$

Lors charge l'énoncé d'une certaine valeur affective, liée aussi bien à le qu'à heure ${ }^{32}$. Ainsi peut-il réaliser une transition entre l'extériorité de l'enchânement narratif et l'intériorité du point de vue - d'où son exploitation dans l'articulation très souple $d u$ discours au récit :

(32) Quant l'empereur vit la vaillance de cestes gens, et que l'un parti ou l'autre failloit que rompist, alors s'escria et dist: " Helas! Ou estoit mon cuer, de souffrir un tel inconvenient? " Lors hastivement gecta sa flesche en disant: " Ho! " Lors fust demandé dont il venoit et qu'il demandoit. (Antoine de la Sale, Jean de Saintré : $267 ; 1451)$

50 Cette aptitude connective tient à une indétermination sémantique basique ${ }^{33}$. Lors a, de fait, un sémantisme indéfini qui demande à être saturé par le cotexte, ce qui favorisera d'autant plus son déclin en tant que forme adverbiale autonome ${ }^{34}$. Le processus d'extension des emplois de lors débouche sur sa réanalyse en un élément de base de locution conjonctive. Lors a une attache privilégiée avec le mot que, dont il complète la «morphologie intégrée ». Cette évolution se perçoit à travers le phénomène de coalescence qui affecte lors que $e^{35}$.

51 En ce sens donc la restructuration grammaticale opérée se base sur des réinterprétations sémantiques des composantes de la locution conjonctive.

52 C'est aussi grâce à de nouvelles acquisitions sémantiques ${ }^{36}$ que lorsque perdure :

\begin{tabular}{|l|l|l|l|l|l|}
\hline & XVI ème $^{\text {èn }}$ & XVII $^{\text {ème }}$ & XVIII $^{\text {ème }}$ & XIX $^{\text {ème }}$ & XX $^{\text {ème }}$ \\
\hline
\end{tabular}




\begin{tabular}{|l|l|l|l|l|l|}
\hline Nombre de textes & 154 & 572 & 554 & 958 & 1633 \\
\hline Nombre de mots & 5831351 & 21840540 & 35396617 & 69795245 & 99840541 \\
\hline Nombre d'occurrences de lorsque & 871 & 11128 & 22164 & 29276 & 33946 \\
\hline Fréquence relative de lorsque $^{37}$ & 149 & 510 & 629 & 419 & 340 \\
\hline
\end{tabular}

Comme le montrent les taux de fréquence relative, lorsque étend son emploi du XVI ${ }^{\text {ème }}$ au XVIII ${ }^{e ̀ m e}$ siècle puis régresse, sans pourtant disparaitre complètement de la langue. Lorsque possède, de fait, un éventail particulièrement large d'emplois et d'effets de sens.

\subsection{Emplois et effets de sens de lorsque}

Le fonctionnement de lorsque ne semble pas déroger au fonctionnement général de lors. Il a, lui aussi, vocation à récupérer dans son entourage phrastique des éléments notionnels destinés à caractériser le procès auquel il se rapporte. Ainsi peut-il être apposé à une indication temporelle, comme en témoignent les exemples suivants :

(33) (...) et il fut obligé de remettre cette lecture à un autre tems, lorsque s'estant plus exercé dans la parole de Dieu, il auroit plus d'ouverture pour lire ce saint prophete. (Mabillon, Traité des études monastiques : $163 ; 1691$ )

(34) Le second jour de cette semaine fut le lendemain matin, lorsque Jésus-Christ venant de Béthanie à la ville eut faim, desseicha le figuier et nettoya le temple de voleurs, comme il les appelle. (Bossuet, Méditations sur l'Évangile : 167 ; 1704)

(35) Le soir, lorsque Claude accompagna Sandoz à la gare, ce dernier lui dit: " A propos, je comptais te faire une confidence ...Je crois que je vais me marier». Du coup, le peintre éclata de rire. (Zola, L'œuvre : $70 ; 1886$ )

(36) Mais hier surtout, lorsque j'ai décidé l'abandon de ma fortune, ce fut une profonde jouissance. (Mauriac, La Fin de la Nuit : 94 ; 1968)

Il présuppose l'existence d'un contexte antérieur qui prépare ou amorce le procès qu'il dénote. Celui-ci intervient en tant que «moment-occurrence ${ }^{38}$ défini-identifié, qui sert de cadre au procès principal :

(37) Lorsque le Roi, par haut désir, et cure

Délibéra d'aller vaincre ennemis,

Et retirer de leur prison obscure

Ceux de son ost à grands tourments soumis,

Il envoya ses fourriers en Judée

Prendre logis sur place bien fondée (...) (Marot, L'Adolescence clémentine : 272-273 ;

1538)

ou en tant que « moment possédant une certaine propriété » :

(38) Saint Luc en tira bien sa part,

Pendant qu'il eust voix en chapitre,

Lorsque la force sert de tiltre,

Chascun met sous le pied les loix. (L'Estoile, Registre-journal du regne de Henri III :

t. $5: 100-101 ; 1587)$

Lorsque possède la capacité de transformer le procès qu'il introduit en un intervalle compact, une période indivise à l'intérieur de laquelle il est impossible de pénétrer, d'où il ressort que son espace temporel, actualisé par le verbe qu'il introduit, ne peut être perçu que comme un tout global, impénétrable à l'analyse : 
(39) La mémoire du déluge étant encore si fraîche parmi les hommes, lorsque Noé vivait encore, Dieu fit ses promesses à Abraham, et lorsque Sem vivait encore, Dieu envoya Moïse, etc. (Pascal, Pensées : 300-301; 1662)

Aussi peut-il marquer la précédence logique (fondée iconiquement sur la précédence temporelle) :

(40) Lorsque tout fut couchiez, ledict bourgeois en habit de femme, comme dit est, entra et trouva ce maistre chanoine en beau hocqueton de camelot, acoustrez comme ung petit esmerillon et qui faisoit tant du miste et de l'honneste que merveille. (Vigneulles, Les Cent Nouvelles nouvelles : $100 ; 1515)$

Sa valeur temporelle peut aussi se teinter d'une valeur adversative, basée sur l'existence d'un décalage entre ce qui correspond à une norme (commune à plus d'un) et ce qui est effectivement validé :

(41) C'est qu'on se croit parjure, lorsqu'on n'est qu'abusé. (Musset, cité par Guilbert,

Lagane \& Niobey (1986, t. $4: 3112)$

ou encore d'une nuance causale :

(42) Allons, allons ! Maitre, cher ami... lorsque tant de malheureux vous bénissent !

(Bernanos, La Joie : 636 ; cité par Imbs $(1983$, t. 10 :1370) ; 1929)

Lorsque semble articuler la représentation factuelle des procès (leur localisation temporelle) et leur représentation intersubjective, et marquer ainsi une relation oppositive ou causale entre les procès simultanés. Il impose, de fait, un certain niveau d'implication du locuteur, ce qui constitue sa coopération à l'interprétation de son environnement discursif.

\section{Conclusion : grammaticalisation ou pragmaticalisation ? $^{39}$}

2 L'émergence de lors que manifeste bien certaines tendances évolutives décrites dans la théorie de la grammaticalisation, et notamment l'utilisation du matériel lexical dans la formation d'un morphème grammatical. Ponctuellement, on peut, par contre, remettre en question l'unidirectionnalité « du moins grammatical » au « plus grammatical », si tant est que l'on ne puisse définir une telle échelle entre adverbe et locution conjonctive.

3 L'histoire de lors que permet d'illustrer aussi l'idée que l'évolution est un processus, n'impliquant pas de changements brusques. Nous pensons que tous les types d'évolution que nous venons de distinguer peuvent être subsumés sous l'étiquette commune de grammaticalisation. Tous ces faits de changements ont, en effet, des points communs, et notamment le caractère progressif de leur évolution. Nous avons remarqué, d'une part, que certaines formes demeurent peu de temps et finissent par disparaître, alors que lorsque perdure et acquiert des sens variés. D'autre part, nous avons constaté que les processus qui déterminent l'évolution de lorsque sont de nature systématique et se font dans un certain ordre. Il s'est avéré, par contre, que l'évolution sémantique est à la source

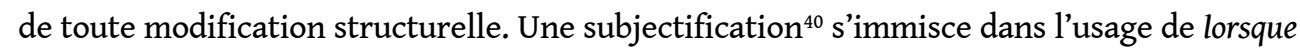
et le modifie de façon récurrente.

64 Faut-il considérer du coup qu'il s'agit d'un phénomène de pragmaticalisation?

Il semble que les valeurs notionnelles de lorsque soient des réseaux sous-jacents du sens temporel, qui s'acquièrent par ajustements à une empiricité du sens commun traversant des phases distinctes d'intersubjectivité. Ce sont les représentations véhiculées 
par les contenus propositionnels, relatives au dit ou sur l'acte énonciatif lui-même, le dire (Ducrot, 1984) qui déterminent le sens de lors, non sans ambiguïté. Nous avons essayé de montrer que que entre en interaction avec l'adverbe lors, et fournit ainsi un second domaine filtrant le contexte. Lorsque est ainsi prédestiné à jouer un rôle dans la structuration du discours et donc à assumer, en plus de la fonction idéationnelle (ou représentationnelle), une fonction textuelle ${ }^{41}$.

Nous avons essayé de montrer que la locution conjonctive lorsque est de nature anaphorique, qu'elle ne peut s'interpréter que par rapport à un repère lié à l'amont du texte ou donné comme présupposé. Elle réintroduit ainsi la mémoire active du texte et/ ou oriente l'interprète vers les connaissances encyclopédiques, faisant le lien non seulement avec ce qui a été précédemment dit, mais aussi avec ce que tout un chacun est censé savoir. Dans les réalisations discursives, elle permet d'établir des rapports d'opposition, de cause à effet, - avec la part de subjectivité qu'elle charrie sans toutefois se départir de sa valeur temporelle, inscrite dans la langue.

À travers l'étude menée ici, on voit s'esquisser une «nouvelle facette " de la grammaticalisation, qui transcende en quelque sorte l'opposition classique lexical/ grammatical. En faisant le lien entre l'élément de base-lexical et le mot que-grammatical composant la locution conjonctive lorsque, nous avons montré que l'on a affaire à une zone catégorielle intermédiaire. Par ailleurs, la vision multipolaire de la signification que nous avons mise au jour récuse l'étanchéité de la séparation entre unités lexicales et unités grammaticales.

La formation de la locution conjonctive se fait dans et par l'usage. Ses processus s'inscrivent dans des jeux intersubjectifs d'ajustement et de régulation qui n'aboutissent à des points fixes que provisoirement et localement. Aussi grammaticalisation et pragmaticalisation se croisent. Nous pouvons ainsi repenser la frontière entre ces deux concepts, dont la pertinence doit être mise en cause.

\section{BIBLIOGRAPHIE}

\section{Ouvrages et articles}

Badiou-Monferran, C.\& Buchi, E. (2012), « Plaidoyer pour la désolidarisation des notions de pragmaticalisation et de grammaticalisation », Actes du colloque du CMLF (en ligne).

Bat-Zeev Shyldkrot, H. (1987), « Quand, alors que et tandis que : un cas classique d'évolutionsémantique » Romance Notes $\mathrm{n}^{\circ} 28,1: 45-50$.

Ben Hamad, L. (2008), « Les locutions conjonctives en question(s) », Linx $n^{\circ} 59$, Les conjonctions en diachronie : parcours sémantiques, Paris, Université Paris X-Nanterre, pp. 83-93.

Ben Hamad, L. (2012a), « La grammaticalisation : Bilan des études et perspectives de recherche ", Studii de lingvistică $\mathrm{n}^{\circ}$ 2, Faculté des Lettres de l'Université d'Oradea (Roumanie), pp. 5-24. 
Ben Hamad, L. (2012b), « L'émergence des locutions conjonctives de simultanéité en ancien français ", in Actes du troisième Congrès mondial de Linguistique française, Actes en ligne : www.linguistiquefrançaise.org.

Ben Hamad, L. (2013), « Réflexions sur le statut du mot que dans les locutions conjonctives : changement et permanence? ", in Van Acker, M., Boutier, M.-G., Hadermann, P. (éds.), Variation et changement en langue et en discours, Mémoires de la Société Néophilologique de Helsinki, pp. 105-132.

Ben Hamad, L. (2015a), « La disparition de dementres que : corrélat de la variation? » in BadiouMonferran, Cl. \& Verjans, T. (éds.), Disparitions et changements linguistiques, actes du colloque international organisé à Dijon (juin 2011), Paris, Honoré Champion, coll. « Linguistique historique ", pp. 419-434.

Ben Hamad, L. (2015b), «Tandis que, entre langue et discours », L'information grammaticale $\mathrm{n}^{\circ}$ 145, pp. 38-45.

Ben Hamad, L. (2015c), « Sur le prétendu figement des locutions conjonctives : le cas de lorsque et alors que ", Revue de Sémantique et Pragmatique n³8, pp. 121- 147.

Benveniste, E. (1974), Problèmes de linguistique générale, t. 2, Paris, Gallimard.

Borillo, A. (1998), « Les adverbes de référence temporelle comme connecteurs temporels de discours », in Vogeleer, S., Borillo, A., Vetters \& Vuillaume, M. (éds.) : 131-145.

Bougy, C. (2000), « Les connecteurs temporels et l'apparition de lors que dans la langue française ", Syntaxe et sémantique $n^{\circ} 1: 39-78$, Caen, Presses universitaires de Caen.

Combettes, B. (2006), « La grammaticalisation des locutions conjonctives en français : l'opposition que / ce que », Faits de langues n 28 : 95-106, Paris Ophrys.

Combettes, B. (2007), « La formation des locutions conjonctives temporelles : l'opposition que/ce que en moyen français ", in P. Le Goffic (éd.) Les mots en qu- du français, Lexique, Villeneuve d'Ascq, Presses Universitaires du Septentrion, p. 47-67.

Combettes, B. (2008), « La variation que / ce que et la formation des locutions conjonctives en français », Linx n 59, p. 15-31.

Dardel (De), R. (1983), Esquisse structurale des subordonnants conjonctionnels en roman commun, Genève, Droz.

Denis, D. \& Sancier-Chateau, A. (1994) Grammaire du français, Le Livre de Poche.

Dostie, G. (2004). Pragmaticalisation et marqueurs discursifs. Analyse sémantique et traitement lexicographique. Bruxelles : De Boeck \& Duculot.

Gaatone, D. (1981), « Conjonctions et locutions conjonctives en français », Folia Linguistica, $\mathrm{n}^{\circ}$ 14, 1/2 : 195-211, Mouton Publishers.

Garagnon, A.-M., Calas, F. (2002) , La phrase complexe. De l'analyse logique à l'analyse structurale, Paris, Hachette Supérieur.

Graeme-Ritchie, R.-L. (1907), Recherches sur la syntaxe de la conjonction "que" dans l'ancien français depuis les origines de la langue jusqu'au commencement du XIII ${ }^{e ̀ m e}$ siècle, Paris, Champion.

Gross, G. (1988), « Réflexions sur la notion de locution conjonctive », Langue française $\mathrm{n}^{\circ} 77$ : 19-36.

Guiraud, P. (1960), Problèmes et méthodes de la statistique linguistique, Paris, Puf.

Heine, B. \& Reh, M. (1984), Grammaticalization and Reanalysis in African Languages, Hamburg, H. Buske. 
Imbs, P. (1956), Les propositions temporelles en ancien français : la détermination du moment, Paris, les Belles Lettres, Publications de la Faculté des lettres de l'université de Strasbourg.

Jonas, P. (1971), Les systèmes comparatifs à deux termes en ancien français, Bruxelles, Éditions de l’Université de Bruxelles.

Kurylowicz, J. (1965), « The evolution of grammatical categories »,Diogenesn ${ }^{\circ}$ 51: 55-71.

Marchello-Nizia, C. (2006),Grammaticalisation et changement linguistique , champs linguistiques, Bruxelles, De Boeck et Larcier.

Martin, R. \& Muller, Ch. (1964), « Syntaxe et analyse statistique : la concurrence entre le Passé antérieur et le Plus que parfait dans La mort le Roi Artu », Travaux de Linguistique et de Littérature, $\mathrm{n}$ - $1:$ 207-233, Strasbourg, Centre de Philologie et de Littératures Romanes de l'Université de Strasbourg II.

Ménard, P. (1998), Syntaxe de l'ancien français, Bordeaux, Bière.

Pierrard, M. (1998), « Comme "relatif à antécédent” en ancien français : grammaticalisation de la proforme indéfinie ", Travaux de linguistique $\mathrm{n}^{\circ} 36$ :127-146.

Raynaud de Lage, G. (1972), Introduction à l'ancien français, Paris, Société d'édition d'enseignement supérieur.

Rubba, J. (1994), « Grammaticalization as Semantic change: A case Study of Preposition Development », in Pagliuca, W. (éd): 81-101.

Skårup, P. (1975), Les premières zones de la proposition en ancien français, Études romanes de l'université de Copenhague.

Stein, D. \& Wright, S. (1995), Subjectivity and subjectivisation. Linguistic perspectives, Cambridge, Cambridge University Press.

Tesnière, L. (1959), Esquisse d'une syntaxe structurale, Paris, Klincksieck.

Traugott, E.-C. \& König, E. (1991), « The semantics-Pragmatics of grammaticalization revisited », in Traugott, E-C. \& Heine, B. (éds) : 189-218.

Traugott, E.-C. (1995), « Subjectification in gramaticalization » in Stein, D. \& Wright, S. (éds), 31-54.

Traugott, E.-C. \& Dasher, R.-B.(2002), Regularity in semantic change, Cambridge, Cambridge University Press.

Dictionnaires

Bloch, O. \& Wartburg, W. (Von) (1968), Dictionnaire étymologique de la langue française, Quadrige, Puf.

Dauzat, A., Dubois, J. \& Mitterand, H. (1994), Dictionnaire étymologique et historique du français, Paris, Librairie Larousse.

Godefroy, F. (1883), Dictionnaire de l'ancienne langue française et de tous ses dialectes, du IXème au XVème siècle, Paris, (Nendeln/Liechtenstein, Kraus Reprint,1969).

Greimas, A.-J. (1979), Dictionnaire de l'ancien français, Paris, Larousse (réed. 2001).

Guilbert, L., Lagane R. \& Niobey, G. (1986), Grand Larousse de la langue française, 7 vol. , Paris, Larousse 
Imbs, P. (dir.) (1971-1994), Trésor de la langue française. Dictionnaire de la langue du XIXème et du XXème siècle (1789-1960), 16 vol. , Paris, Editions du Centre National de la Recherche Scientifique (t.1-10), Gallimard (depuis le t. 11)

Lafaye, B. (1884), Dictionnaire des synonymes de la langue française, Paris, Librairie Hachette \& Cie http://gallica.bnf.fr.

Wartburg, W. (Von) (1922-...), Französisches Etymologisches Wörterbuch, Eine Darstellung des galloromanischen sorachschatzes, Tübingen, Moh, puis, Basel, Hebing und Lichtenhan.

\section{Bases de données utilisées}

BFM

Base de Français Médiéval. Lyon : UMR5191ICAR/ENS-LSH, http://www.bfm.ens-lsh.fr. DMF

Base du Dictionnaire de Moyen Français, UMR 7118 ATILF / Nancy 2. http://www.atilf.atilf.fr/ dmf.htm.

Frantext

UMR 7118 ATILF/Nancy2, http://www.frantext.fr

\section{NOTES}

1. Nous tenons à remercier Jacques François, Annie Bertin ainsi que les relecteurs anonymes de la revue pour leurs remarques pertinentes.

2. Insistons sur le fait que les résultats obtenus ne sont exhaustifs que par rapport au corpus retenu, constitué des bases BFM, DFM et Frantext.

3. D'après Imbs, l'émergence de ce tour correspond à « l'intervention plus ou moins arbitraire, ou en tout cas personnelle d' (...) un romancier, c'est-à-dire, un auteur qui écrit en octosyllabe. » (op. cit. : 300). Elle est donc due, à son sens, aux exigences techniques et esthétiques de l'octosyllabe. Pour notre part, nous serions plutôt encline à y voir une portée plus large encore.

4. Notons que lués que est dû également à Chrétien de Troyes : a trestoz congié demandai, / si m'en alai lués que je poi. (Chrétien de troyes, Chevalier au Lion ou Yvain, v. 274-275); Lués que Cligés le vit movoir / Et de son non oï le voir. (Chrétien de Troyes, Cligés, v. 4829-4830).

5. Nous empruntons ce terme à Kurylowicz (1949:23).

6. Partant de données empiriques, observables, nous ne pouvons, de fait, reconstruire le processus de constructionalisation de lorsque et restituer les phénomènes en jeu dans cette évolution que par hypothèse.

7. Rappelons que Dardel (1983 : 19) oppose : « locution subordonnante conjointe primaire, qui est formée directement par préfixation, sans passer par une locution subordonnante disjointe et la locution subordonnante conjointe secondaire, formée à partir d'une locution disjointe ».

8. Pour une analyse plus précise, nous nous permettons de renvoyer le lecteur à Ben Hamad (2015a).

9. Benveniste (1974: 126-127) propose de distinguer "les transformations innovantes", qui « changent l'effectif des catégories formelles de la langue » des " transformations conservantes ».

10. Cette hypothèse rencontre l'approbation de Marchello-Nizia (2006: 110) : «Un bon nombre de nouvelles unités introduites dans la grammaire ne sont pas des nouveautés conceptuelles, mais des variantes, des doublons de morphèmes existants, des morphèmes-miroirs, qui parfois vont jusqu'à reprendre et redoubler dans l'expression les formes déjà présentes. ». 
11. La saisie de ce phénomène est délicate car elle suppose des renseignements statistiques. Nous ne pouvons procéder ici à une enquête numérique d'autant que l'intrication des valeurs est particulièrement sensible, rendant incertaine les proportions chiffrées.

12. Notre analyse est proche de celle proposée par Jonas (1971:49) pour les comparatives : «Ce serait une erreur de considérer que dans les systèmes envisagés, com(e) était l'articulant de base, si l'on peut dire, et que les éléments pouvant le précéder s'employaient facultativement et n'avaient d'autres fins que de conférer à la comparaison une dose plus ou moins forte d'expressivité. ».

13. Cette contrainte d'homogénéité s'exerce ainsi sur l'adverbe et le subordonnant en leur imposant la co-orientation.

14. Voir à ce sujet Ben Hamad (2012b)

15. Prêts à différencier, à moduler et à nuancer un noyau conceptuel commun, ces adverbes marquent différemment les formes synthétiques sur lesquelles ils se greffent. Ils font dépendre l'avènement de la simultanéité de leur sens. L'adverbe tant, étudié par Weill (2002), "ancre la situation dans un nouvel état de relation au vrai », or est pour Ollier (1995) "un opérateur de rupture », si, étudié par Marchello-Nizia (1985), est « un marqueur d'assertion, qui fait de la subordonnée un préasserté à la régissante ».

16. Ore se comporte ici comme un nom, comme en latin. (Voir Wartburg, 1922: 467-471). Nous relevons d'autres occurrences de ore qui peuvent être rangées dans cette même catégorie d'emploi. C'est par exemple le cas dans : E establi la tempested de lui en l'ore, / e turent li fluet de lui. $E$ esledecerent pur ce qu'il se turent, /e demena els el port de la volunted d'els. (Libri psalmorum : 165 ; première moitié du XII ${ }^{\text {ème }}$ siècle) ; Quant ore fu d'aler gesir, /L'empereres, si com il dut, /la nuit avoec sa fame jut. (Chrétien de Troyes, Cligès, v. 3290-3292); Quant ore fu, si l'en menerent / Colchier en une chanbre clere, (Chrétien de Troyes, Chevalier au Lion ou Yvain, v. 4010-4011).

17. Ce tour hybride n'a pas de vrais prolongements après cette période archaïque. Nous ne l'avons trouvée attesté, en effet, que dans Le Roman de Thèbes : Li reis de Thèbes bien le sot, / Car en l'ost ses espies ot / Qual hore que cil de l'ost murent, / Et les espies al rei furent (v. 7409-7412).

18. Divers auteurs ont déjà observé que, rattaché à un substantif signifiant un moment, que a une valeur relative aisément discernable. Cf. notamment Imbs (1956 : 217), Raynaud de Lage (1972 : 141), Ménard (1988: 84).

19. Nous utilisons ici cette notion de source afin d'éviter un certain nombre d'écueils. De fait, comme l'a constaté Tesnière (1959: 86-87): "Malheureusement, le terme antécédent a l'inconvénient de désigner le mot en question, non d'après sa nature, qu'on ne cherche même pas, mais d'après sa position qui est sujette à toutes les variations que lui impose l'ordre linéaire de la chaîne parlée.»

20. Au regard de Wartburg (1992: 467-475), or, lors et alors ont le même étymon, le latin hōra, 'heure'. Bloch \& Wartburg $(2002: 374,446)$ précisent, à leur tour, que or (en outre ore, ores) est issu d'un syntagme nominal à l'ablatif : ha hora, altération du latin classique hac hora ‘̀̀ cette heure' et donnent lors (en outre lor et lores), « dont le développement a été parallèle au type qui a donné naissance à or ", comme équivalent de illa hora à cette heure-là'. Greimas (1989) ainsi que Dauzat, Dubois \& Mitterand (1994) adoptent une attitude similaire.

21. Il importe de s'aviser que la quantification ne constitue pas, pour nous, la pierre de touche de toutes nos hypothèses, comme le veulent Martin \& Muller (1964 : 233), par exemple, qui y voient : «(...) des caractéristiques (...) simples (indices, coefficients), véritables instruments de mesure qui permettent une juste appréciation des faits, une épreuve des hypothèses ". L'approche quantitative est mise en œuvre, ici, pour essayer de faire émerger ces hypothèses. Nous n'adhérons pas, non plus, au courant qui fait de la statistique une assise expérimentale, partant du postulat que la linguistique est la science statistique type. Cf. par exemple Guiraud (1960: 15-22). La statistique est, pour nous, un appareil de mesure, qui offrira à notre étude des garanties scientifiques, mais sans jamais la transformer en recherche expérimentale. 
22. La régression des locutions subordonnantes comparatives formées sur com(e), aujourd'hui disparues, en porte témoignage. Cf. Pierrard (1998:127).

23. Il nous semble que ce paramètre, rarement envisagé comme significatif, est au contraire au cœur du problème.

24. Certaines occurrences existent, mais de façon sporadique. Imbs et al. citent par exemple : Seules (...) subsistent les cathédrales, désaffectées et muettes (...) lors des artistes, séduits (...), veulent en refaire pour une heure le théâtre du drame mystérieux qui s'y déroulait (Proust, Pastiches et mélanges, 1919, p. 198)

25. Sur ce point, cf. notamment Marchello-Nizia (2006:110).

26. Observons cependant que, selon Imbs (1956: 209), lors est bâti non sur un nom temporel du latin mais sur un adverbe médiéval. Il voit dans le désir d'expressivité du locuteur le facteur déclenchant l'intégration de ce nouveau morphème dans la grammaire : « (...) très tôt la langue a créé pour l'expression de la coïncidence temporelle (ou présent "relatif") un morphème spécifique, obtenu à partir de or par adjonction de l'article l'. ». Nous ne pouvons souscrire à cette opinion. Il est de fait difficile de considérer la création d'un nouvel adverbe comme le simple résultat d'une évolution morpho-phonétique, motivée par une prédilection marquée pour l'expressivité.

27. Sur ce point, cf. en particulier Stein et Wright (1995).

28. C'est ce rôle d'anaphorique qui permet - on le verra - l'articulation des valeurs temporelles et notionnelles.

29. Nous empruntons les termes de zone annexe et place de fondement à Skårup (1975).

30. Certaines études ont essentiellement insisté sur le fait que cet adverbe introduit dans le récit des événements un supplément de cohérence, de signification. Il est ainsi considéré comme une particule narrative qui contribue à en constituer la cohésion et en assurer le flux. Ainsi Sakari (1997 : 361) écrit : «Situant le procès dans la chaîne des événements racontés, l'adverbe lors ponctue le récit et en assure une certaine cohérence ».

31. Comme le remarque Ménard (1998: 183), à qui est emprunté cet exemple, « lors détache d'une manière dramatique un moment important de l'action", à la différence de atant et adont qui «ont généralement une valeur assez neutre ».

32. Pour le détail, voir Ben Hamad (2015c).

33. Nous ne développerons pas ce point ici. Cf. Ben Hamad (2015c).

34. La décadence de l'adverbe lors n'est cependant pas complète. Il s'emploie en couplage avec les prépositions dès et depuis, comme le note Borillo (1998:141).

35. Certains auteurs considèrent lorsque comme une conjonction simple. Cf. notamment, Denis \& Sancier-Chateau (1994: 143) et Garagnon \& Calas (2002 : 75).

36. Heine \& Reh (1984) parlent, en l'occurrence, d'« expansion ». On trouve encore des termes comme «strengthening of informativeness » chez Traugott et König (1991), « semantic gain » chez Rubba (1994) ou encore de « strengthening of speaker perspective » chez Traugott (1995).

37. Les chiffres présentés ici ont été obtenus grâce au calcul proportionnel suivant: le nombre d'occurrences relevées de lorsque divisé par le nombre total des mots et multipliée par 1.000.000.

38. Ceci expliquerait que le moment indiqué par lorsque soit considéré comme plus précis que celui indiqué par quand. Cf. notamment Lafaye (1884) : «Quand est général, vague, (...) Au contraire, lorsque est précis, (...). Dans les propositions générales, (...) où on parle d'une manière absolue, indépendamment des cas ou des événements particuliers, quand est le seul mot qui convienne (...). Mais dans les propositions particulières, où il s'agit de ce qui s'est effectivement passé, où l'on raconte, c'est lorsque qui doit être préféré. ».

39. Il n'existe pas vraiment de consensus sur ce que recouvrent les concepts de grammaticalisation et de pragmaticalisation. Certains (Traugott \& Dasher 2002, Dostie 2004, entre autres) considèrent que la pragmaticalisation est un mécanisme sous-jacent à la 
grammaticalisation. D'autres plaident, par contre, en faveur d'une désolidarisation totale des deux notions (Cf.notamment Badiou-Monferran \& Buchi, 2012).

40. D'après Traugott (1995: 35), «Subjectification refers to a pragmatic-semantic process whereby 'meanings become increasingly based in the speaker's subjective belief state attitude toward the proposition. ".

41. Nous ne détaillerons pas ce point ici. (Cf. Ben Hamad, 2013)

\section{RÉSUMÉS}

Nous avons pour ambition de fournir une analyse longitudinale de l'émergence et de l'évolution des locutions conjonctives sur plus d'un millénaire de langue française. Nous nous penchons ici sur le cas de lorsque. Nous tentons d'en étudier d'abord la genèse. Nous procédons ensuite à décrire les parcours qu'il a pu suivre, de l'ancien français jusqu'au français moderne, en essayant de découvrir les processus qui y sont impliqués.

We aim to provide a longitudinal analysis of the emergence and the evolution of conjunctive phrases in more than a thousand years of the French language. Here we look at the case of lorsque . We first try to study its genesis. Then we proceed to describe the paths it follows, from the old French to the modern French, trying to discover the processes involved.

\section{INDEX}

Keywords : Keywords : emergence, evolution, change, variation, process

Mots-clés : émergence, évolution, changement, variation, processus

\section{AUTEUR}

\section{LEÏLA BEN HAMAD}

Université de Sousse, Tunisie

LDC, MoDyCo 